\title{
Fostering precision psychiatry through bioinformatics
}

\author{
Brisa S. Fernandes, ${ }^{1}$ (D) João Quevedo, ${ }^{2}$ (iD Zhongming Zhao ${ }^{1,2,3}$ (D) \\ ${ }^{1}$ Center for Precision Health, School of Biomedical Informatics, The University of Texas Health Science Center at Houston (UTHealth), \\ Houston, TX, USA. ${ }^{2}$ Translational Psychiatry Program, Faillace Department of Psychiatry and Behavioral Sciences, McGovern Medical School, \\ UTHealth, Houston, TX, USA. ${ }^{3}$ Human Genetics Center, School of Public Health, UTHealth, Houston, TX, USA.
}

\begin{abstract}
"No tool is omnicompetent.
There is no such thing as a master-key that will unlock all doors."

Arnold Toynbee, A Study of History
\end{abstract}

Precision medicine is changing the way in biomedical research and healthcare, thanks to the rapid advancement of biotechnologies (e.g., whole-genome sequencing), electronic health records (EHR), and informatics, among others. Precision psychiatry is a recognized priority that represents a paradigm shift in how psychiatry was traditionally conceptualized until just a few years ago. It preaches that the "right drug to the right patient at the right time" approach will translate into better and more durable clinical benefits. However, how can we achieve this lofty goal? This, as can be pedestrianly put, is easier said than done. By now, it has become abundantly clear that, due to the intrinsic complexity of psychiatry and its disorders, no single biomarker will be capable of diagnosing or predicting with sufficient accuracy that translates into meaningful clinical utility. ${ }^{1}$

Historians have long theorized that civilizations are born from more primitive societies as a response to challenges and penalization, and that civilizations develop in different ways due to their different approaches to the challenges they face. ${ }^{2}$ We can easily theorize that the same is true in science; precision psychiatry emerged as a new paradigm as a response to the challenge of improving outcomes and ameliorating the suffering of those afflicted by mental disorders, and by the penalties that we will incur if we fail to develop it. However, how precision psychiatry will be developed rests on us and the approach that we take to overcome the challenge of discovering and developing biomarkers. The large field of bioinformatics is a relatively new intersection in psychiatry, and provides not only a single tool to discover and develop new biosignatures and drugs that optimally match them, ${ }^{3}$ but provides a sizeable armamentarium of tools that, in combination, can assist in unlocking the elusive goals of precision psychiatry.

Due to the fact that no single cause is responsible for a given psychiatric disorder and that their pathophysiology most probably arises from a myriad of combined factors,

Correspondence: Zhongming Zhao, Center for Precision Health, School of Biomedical Informatics, The University of Texas Health Science Center at Houston, Houston, TX 77030, USA.

E-mail: zhongming.zhao@uth.tmc.edu

Submitted Jun 19 2021, accepted Jun 20 2021, Epub Aug 092021. bioinformatics and systems-biology data-driven approaches, coupled with artificial intelligence, are exciting and have become a burgeoning strategy in the last decade, mainly in genomics. ${ }^{4}$ However, psychiatric disorders are not only polygenic by nature but also environmentally nurtured to a large extent. The latter suggests that genomics in isolation will not be enough; in that spirit, the aggregation of multi-omics data, such as transcriptomics, proteomics, and metabolomics, among others, which captures different although complementary levels of information which, when integrated, can lead to identification of pathways with stronger biological meaning, is necessary. Accordingly, it has been a major focus of work by bioinformaticians, and significant progress has been made on that front, although much remains to be done. ${ }^{5,6}$ Numerous high-throughput omics studies have been conducted, providing an accumulated catalog of susceptible variants and genes in several disorders; however, results have been highly heterogeneous. The variants and genes nominated by different genetics and omics studies, such as genotyping data, copy number variations, de novo mutations, epigenetics, and transcriptomics, often have limited overlap with each other. To assist with this challenge, we developed a method named mega-analysis of odds ratio (MegaOR), with capabilities of prioritizing genes and providing multi-dimensional evidence. ${ }^{7}$ Even when the task at hand is analyzing a single modality in isolation (for instance, genomics), the need to aggregate distinct datasets to draw firmer conclusions poses a challenge. Developing techniques that allow such integration and harmonization has been a focus of many bioinformaticians. ${ }^{8}$ Finally, in psychiatry, we face a drought when it comes to discovering new drugs to treat these highly debilitating disorders; developing new drugs is a very long and hazardous process, and most developed compounds later fail when tested in clinical trials. On this front, bioinformatics also poses as an attractive solution; by using in silico techniques, we can de-risk what is otherwise a highly risky and lengthy business by discovering and selecting optimal molecular targets for repurposing currently existing drugs that will perfectly match individual patients with a given molecular profile, thus improving treatment outcomes, the ultimate

How to cite this article: Fernandes BS, Quevedo J, Zhao Z. Fostering precision psychiatry through bioinformatics. Braz $\mathrm{J}$ Psychiatry. 2022;44:119-120. http://dx.doi.org/10.1590/1516-44462021-2083 
goal of precision psychiatry. In this sense, pharmacogenomic tests have started this field, but we still have a long way to go. ${ }^{1,9,10}$

"No tool is omnicompetent. There is no such thing as a master-key that will unlock all doors."2 Bioinformatics, alone, will not unlock all doors, but it is a vast and versatile field with many tools which have the potential of unlocking many of the necessary doors for the development of precision medicine in psychiatry and, as such, shows great promise for the discovery of biomarkers and new pharmacological treatments.

\section{Disclosure}

The authors report no conflicts of interest.

\section{References}

1 Fernandes BS, Williams LM, Steiner J, Leboyer M, Carvalho AF, Berk M. The new field of 'precision psychiatry'. BMC Med. 2017;15:80.

2 Toynbee A. A study of history. London: Oxford University Press; 1951.
3 Tenenbaum JD, Bhuvaneshwar K, Gagliardi JP, Hollis KF, Jia P, $\mathrm{Ma} \mathrm{L}$, et al. Translational bioinformatics in mental health: open access data sources and computational biomarker discovery. Brief Bioinform. 2019;20:842-56.

4 Shi X, Zhao Z, Wang K, Shen L. The International Conference on Intelligent Biology and Medicine (ICIBM) 2020: data-driven analytics in biomedical genomics. BMC Med Genomics. 2020;13:189.

5 Ressler KJ, Williams LM. Big data in psychiatry: multiomics, neuroimaging, computational modeling, and digital phenotyping. Neuropsychopharmacology. 2021;46:1-2.

6 Paczkowska M, Barenboim J, Sintupisut N, Fox NS, Zhu H, Abd-Rabbo D, et al. Integrative pathway enrichment analysis of multivariate omics data. Nat Commun. 2020;11:735.

7 Jia P, Chen X, Xie W, Kendler KS, Zhao Z. Mega-analysis of odds ratio: a convergent method for a deep understanding of the genetic evidence in schizophrenia. Schizophr Bull. 2019;45: 698-708.

8 Jia P, Pei G, Zhao Z. CNet: a multi-omics approach to detecting clinically associated, combinatory genomic signatures. Bioinformatics. 2019;35:5207-15.

$9 \mathrm{Hu} \mathrm{R}, \mathrm{Xu} \mathrm{H}$, Jia P, Zhao Z. KinaseMD: kinase mutations and drug response database. Nucleic Acids Res. 2021;49:D552-61.

10 Jia P, Hu R, Pei G, Dai Y, Wang YY, Zhao Z. Deep generative neural network for accurate drug response imputation. Nat Commun. 2021;12:1740. 